\title{
FAMILIES OF FINITE CHARACTER AND ESSENTIAL VALUATIONS $\left(^{1}\right)$
}

\author{
BY \\ MALCOLM GRIFFIN $\left({ }^{2}\right)$
}

1. Introduction. A family $\Omega$ of valuations of the field $K$ is said to be of finite character if for every $x \in K, x \neq 0$, the set $\{w \in \Omega \mid w(x) \neq 0\}$ is finite. If $w \in \Omega$ has ring $\mathscr{R}_{w}$ and maximal ideal $\mathscr{P}$ then the ring $A=\bigcap_{w \in \Omega} \mathscr{R}_{w}$ is said to be defined by $\Omega$ and $\mathscr{P} \cap A$ is a prime ideal called the centre of $w$ on $A$, denoted $Z(w)$. If $\mathscr{R}_{w}=A_{Z(w)}$ then $w$ is said to be an essential valuation for $A$.

A ring defined by a family of finite characters consisting of essential valuations is called a ring of Krull type. The properties of such rings are investigated in [2].

In this paper we indicate necessary and sufficient conditions in order that the ring defined by a family of finite character be a ring of Krull type. We recall that in [3] Krull showed that if $\Omega$ is a family of finite character consisting of rationalvalued rank one valuations, then $A=\bigcap_{w \in \Omega} \mathscr{R}_{w}$ may also be defined by a subfamily of $\Omega$, consisting of essential valuations. Krull then posed the question: Is this still the case for families of finite character of arbitrary rank one valuations? As an application of our theory, we provide an example to show that the answer to the above question is negative.

2. Essential valuations and uniformly critical well centred families. Let $\Gamma$ be a totally ordered group. A subgroup $\Delta$ of $\Gamma$ is called an isolated subgroup if $\delta \in \Delta$ and $\delta \geqq \gamma \geqq 0$ imply that $\gamma \in \Delta$.

If $w, w^{\prime}$ are valuations of $K$ with groups $\Gamma, \Gamma^{\prime}$ and rings $\mathscr{R}, \mathscr{R}^{\prime}$ with $\mathscr{R} \subseteq \mathscr{R}^{\prime}$, we say that $w^{\prime}$ is coarser than $w$ and write $w^{\prime} \leqq w$. If $w^{\prime} \leqq w$, then for some prime ideal $\mathscr{P}$ of $\mathscr{R}, \mathscr{R}^{\prime}=\mathscr{R}_{\mathscr{P}}$ and $\Gamma^{\prime} \cong \Gamma / \Delta$ where $\Delta$ is the isolated subgroup generated by those elements of $\Gamma$ which do not belong to $\mathscr{P}$.

If the valuation is such that its group has no minimal nonzero isolated subgroup we say that $w$ is a limit valuation.

Let $\Omega$ be a family of valuations defining $A$. Let $x \in K, x \neq 0$ and let $P$ be a prime ideal of $A$; we use the following notation:

$\Omega(x)=\{w \in \Omega \mid w(x) \neq 0\}, \Omega^{+}(x)=\{w \in \Omega \mid w(x)>0\}, \Omega^{-}(x)=\{w \in \Omega \mid w(x)<0\}$, $\omega_{-}(P)=\{v$ valuation of $K \mid Z(v) \subseteq P$ and there exists $w \in \Omega$ such that $v \leqq w\}$,

Received by the editors February 22, 1966.

(1) Part of the research done for a Ph.D degree at Queen's University, Kingston, Ontario, under the direction of Dr. Paulo Ribenboim, with financial assistance from the National Research Council of Canada.

$\left({ }^{2}\right)$ Applied Mathematics Division, Department of Scientific and Industrial Research, Wellington, N.Z. 
$\omega_{0}(P)=\{v$ valuation of $K \mid Z(v)=P$ and there exists $w \in \Omega$ such that $v \leqq w\}$,

$\Omega_{0}(P)=\left\{w \in \Omega \mid\right.$ there exists $v \in \omega_{0}(P)$ such that $\left.w \geqq v\right\}$,

$\Omega_{+}(P)=\{w \in \Omega \mid Z(w) \supseteq P\}$.

If $\Omega$ is of finite character and $P \neq 0$, all these families except $\omega_{-}(P)$ must be finite.

Given $x \in K, x \neq 0$, let $w$ be a valuation of $K$ with group $\Gamma$, such that $w(x)<0$.

Let $\Delta$ be the largest isolated subgroup of $\Gamma$ such that $w(x) \notin \Delta$. The valuation $v$, coarser than $w$, with value group $\Gamma / \Delta$, is called a singular valuation at $x$ determined by $w$. A singular valuation is not a limit valuation since $v(x)$ generates a minimal nonzero subgroup of $\Gamma / \Delta . Z(v)$ is called a singular prime of $x$.

Let $\Sigma(x)$ denote the set of singular valuations at $x$, determined by all valuations $w \in \Omega^{-}(x)$; let $M(x)=\left\{v \in \Sigma(x) \mid Z(v)\right.$ is minimal among those $Z\left(v^{\prime}\right)$ such that $\left.v^{\prime} \in \Sigma(x)\right\}$; we note that if $\Omega$ is a family of finite character, then $M(x)$ is finite, not empty, for every $x \notin A$.

The valuations belonging to $M(x)$ are said to be critical at $x$ (for the ring $A$ ). A prime ideal $P$ of $A$ is said to be a critical prime for $x$ if it is the centre of a critical valuation at $x$. We say that $w \in \Omega$ is uniformly critical (for $A$ ) if each valuation coarser than $w$ which is not a limit valuation is a critical valuation (at some $x \in K$, $x \neq 0$ ).

We say that a valuation $v$ with group $\Gamma$ and ring containing $A$ is well centred on $A$ if for each $\gamma \in \Gamma^{+}$there exists $a \in A$ such that $v(a)=\gamma$. We say that a valuation $v$ coarser than some valuation of $\Omega$ is strongly well centred on $A$ if $v^{\prime}$ is well centred for each $v^{\prime} \in \omega_{0}(Z(v))$.

Lemma 1. Let $\Omega$ be a family of valuations defining $A$. If $w \in \Omega$ is an essential valuation then it is uniformly critical and well centred.

Proof. Let $v, v \leqq w$, be a valuation which is not a limit valuation; then there exists a finest valuation $v^{\prime}$ which is strictly coarser than $v$, (it is the valuation with group $\Gamma_{v} / \Delta$ where $\Delta$ is the minimal isolated subgroup of $\Gamma_{v}$ ). Then there exists $x \in \mathscr{R}_{v^{\prime}}$ such that $x \notin \mathscr{R}_{v}$ so that $v \in \Sigma(x)$.

Suppose that $v^{\prime \prime} \leqq w^{\prime \prime} \in \Omega$ with $v^{\prime \prime}(x)<0$ and $Z\left(v^{\prime \prime}\right) \subseteq Z(v)$. Then $A_{Z\left(v^{\prime \prime}\right)} \supseteq A_{Z(v)}$ $\supseteq A_{Z(w)}=\mathscr{R}_{w}$; hence $A_{Z\left(v^{\prime \prime}\right)}$ is a valuation ring, and $v^{\prime \prime}$ is essential; from $v^{\prime \prime} \leqq w$ it follows that $v \leqq v^{\prime \prime}$ or $v^{\prime \prime} \leqq v$. In the first case, $Z(v) \subseteq Z\left(v^{\prime \prime}\right)$; hence $A_{Z(v)} \supseteq \mathscr{R}_{v^{\prime \prime}}$; so $v=v^{\prime \prime}$. In the second case, since $v \in \Sigma(x)$ and $v^{\prime \prime}(x)<0$ it follows that $v=v^{\prime \prime}$ and so $Z(v)=Z\left(v^{\prime \prime}\right)$ and $v$ is critical. Thus $w$ is uniformly critical.

Let $\gamma \in \Gamma_{w}^{+}$. Then there exists $x \in \mathscr{R}_{w}$ such that $w(x)=\gamma$. Since $\mathscr{R}_{w}=A_{z(w)}$ there exist $a, b \in A$ with $b \notin Z(w)$ such that $x=a / b$. Then $w(a)=w(a)-w(b)=\gamma$.

LemMA 2. Let $P$ be a critical prime at $x$ and $v \in \Sigma\left(a x^{n}\right)$ with $a \in A, n \geqq 1$ integer, and $Z(v) \subseteq P$. Then $Z(v)=P$.

Proof. Since $v \in \Sigma\left(a x^{n}\right), v\left(x^{n}\right)<-v(a) \leqq 0$ so that $v(x)<0$ and there exists $v^{\prime} \leqq v$ with $v^{\prime} \in \Sigma(x)$ and $Z\left(v^{\prime}\right) \subseteq Z(v) \subseteq P$. Since $P$ is a critical prime for $x$ it follows that $Z\left(v^{\prime}\right)=Z(v)=P$. 
REMARK. If $v$ is well centred, and $v^{\prime}<v$ then $Z\left(v^{\prime}\right) \subset Z(v)$; for $\Gamma_{v^{\prime}} \cong \Gamma_{v} / \Delta$ where $\Delta \neq(0)$ and if $0<\delta \in \Delta$, then because $v$ is well centred there exists $a \in A$ with $v(a)=\delta$, but $v^{\prime}(a)=0$ and so $a \in Z(v), a \notin Z\left(v^{\prime}\right)$.

Lemma 3. Let $\Omega$ be a family of finite character defining $A$. Let $P$ be a prime ideal of $A$. For every $x \in K, x \neq 0$, there exists $a \in A, a \notin P$ such that if $w \in \Omega^{-}(a x)$ then there exists $v \in \Sigma(x)$ with $Z(v) \subseteq P$, such that $w \geqq v$. In particular, if $P$ contains no singular prime of $x$ then ax $\in A$.

Proof. Let $\Omega_{1}=\{w \in \Omega \mid$ there exists $v \in \Sigma(x)$ such that $Z(v) \subseteq P$ and $w \geqq v\}$. Since $\Omega$ is of finite character there exists an element $a \in A, a \notin P$, for which the set $\Omega_{2}$ of valuations in $\Omega^{-}(a x)$ not contained in $\Omega_{1}$ is minimal.

If $w_{1} \in \Omega_{2}$, then $w_{1} \in \Omega^{-}(a x)$ so $w_{1} \in \Omega^{-}(x)$, and there exists $v_{1} \leqq w_{1}$ with $v_{1} \in \Sigma(x)$ and $Z\left(v_{1}\right) \nsubseteq P$. Let $a_{1} \in Z\left(v_{1}\right), a_{1} \notin P$. If $w_{1}\left(a_{1}^{n} x\right) \leqq 0$ for all $n$ then $n w_{1}\left(a_{1}\right) \leqq-w_{1}(x)$ and $w_{1}\left(a_{1}\right)$ belongs to the largest isolated subgroup $\Delta_{1}$ of $\Gamma_{w_{1}}$ not containing $w_{1}(x)$, which implies $v_{1}\left(a_{1}\right)=0$, a contradiction to $a_{1} \in Z\left(v_{1}\right)$. It follows that for some $n$, $w_{1}\left(a_{1}^{n} x\right)>0$. Therefore $\Omega^{-}\left(a_{1}^{n} a x\right)-\left[\Omega_{1} \cap \Omega^{-}\left(a_{1}^{n} a x\right)\right] \subseteq \Omega_{2}-\left\{w_{1}\right\}$; because if $w \in \Omega$, $w\left(a_{1}^{n} a x\right)<0$, then $w(a x) \leqq w\left(a_{1}^{n} a x\right)<0$, on the other hand $w \neq w_{1}$ since $w_{1}\left(a_{1}^{n} a x\right) \geqq 0$. This is a contradiction to the minimality of $\Omega_{2}$; we conclude that our hypothesis $w_{1} \in \Omega_{2}$ must have been incorrect, i.e., $\Omega_{2}=\varnothing$, and there exists $a \in A, a \notin P$ such that $w \in \Omega^{-}(a x)$ implies $w \in \Omega_{1}$. In the particular case, $\Omega_{1}$ is empty, hence $a x \in A$.

Lemma 4. Let $\Omega$ be a family of finite character defining $A$. Let $x \in K, x \neq 0$, and let $v_{1} \in M(x)$ be strongly well centred. Then

(i) $Z(v) \subseteq Z\left(v_{1}\right)$ implies $v(x) \leqq 0$;

(ii) $Z(v) \subseteq Z\left(v_{1}\right)$ and $v(x)<0$ if and only if $Z(v)=Z\left(v_{1}\right)$.

Proof. Let $Z\left(v_{1}\right)=P, M(x) \cap \omega_{0}(P)=\left\{v_{1}, \ldots, v_{n}\right\}$. Since $v_{1}$ is well centred there exists $a_{1} \in A$ such that $v_{1}\left(a_{1}\right)=-v_{1}(x)>0$, i.e. $v_{1}\left(a_{1} x\right)=0$.

Either $v_{i}\left(a_{1} x\right) \geqq 0, i=1, \ldots, n$, or there exists $v_{i}$, say $v_{2}$, such that $v_{2}\left(a_{1} x\right)<0$. Since $v_{2}$ is well centred, there exists $a_{2} \in A$ such that $v_{2}\left(a_{2}\right)=-v_{2}\left(a_{1} x\right)>0$, i.e., $v_{1}\left(a_{2} a_{1} x\right) \geqq 0, v_{2}\left(a_{2} a_{1} x\right)=0$.

We may repeat the argument, if necessary. Then we will find $r, 1 \leqq r \leqq n$, and $a=a_{r} a_{r-1} \cdots a_{2} a_{1} \in A$ such that $v_{i}(a x) \geqq 0$ for all $i=1, \ldots, n$ while $v_{r}(a x)=0$.

Now $v \in \Sigma(a x)$ implies $Z(v) \nsubseteq P$. Indeed if $Z(v) \subseteq P$, and $v \in \Sigma(a x)$ then $v \in \Omega^{-}(x)$; let $v^{\prime} \leqq v, v^{\prime} \in \Sigma(x)$ and $Z\left(v^{\prime}\right) \subseteq Z(v) \subseteq P$, then by Lemma $2, Z\left(v^{\prime}\right)=Z(v)=P$; since $v$ is well centred, by the remark we have $v^{\prime}=v$, hence $v \in \Sigma(x)$; the same argument shows also that $v \in M(x)$. Hence, $v=v_{i}$ for some $i, 1 \leqq i \leqq n$, in contradiction to $v_{i}(a x) \geqq 0$.

Thus by Lemma 3 there exists $b \in A, b \notin P$ such that bax $\in A$. Since $v_{r}($ bax $)$ $=v_{r}(a x)=0$ we have $b a x \notin P$.

(i) If $Z(v) \subseteq P=Z\left(v_{1}\right)$ then $v(b a x)=0$, so $v(x)=-v(b a) \leqq 0$.

(ii) If $v(x)<0$ with $Z(v) \subseteq P$, then by Lemma $2, Z(v)=P=Z\left(v_{1}\right)$. Conversely, if $Z(v)=P$ then since $a \in P, v(a)>0$, hence $v(x)=-v(b a)<0$. 
COROLlary. Let $P$ be a critical prime at $x \in K, x \neq 0$ such that all the valuations of $\omega_{0}(P)$ are well centred. Then there exists an element $a \in K, a \notin P$, such that $\Omega^{-}(a x)$ $=\Omega_{0}(P)$.

Proof. Let $v^{\prime} \in M(x)$ be such that $Z\left(v^{\prime}\right)=P$. By Lemma 3, there exists $a \in A$, $a \notin P$, such that if $w \in \Omega^{-}(a x)$ then there exists $v \in \Sigma(x)$, with $Z(v) \subseteq P, v \leqq w$. If $w \in$ $\Omega^{-}(a x)$ then $w \in \Omega_{0}(P)$ since by Lemma 2 we must have $Z(v)=P$. On the other hand, if $w \in \Omega_{0}(P)$ there exists $v$ such that $Z(v)=P, v \leqq w$; by Lemma $4, v(x)<0$ and since $a \notin P$ we have $v(a x)<0$, and so $w(a x)<0$; therefore $w \in \Omega^{-}(a x)$.

We now show how to define, for a given prime $P$ and $w \in \Omega_{+}(P)$, the coarsest valuation $v \leqq w$ with centre containing $P$; we denote this valuation $C_{P}(w)$.

Let $\Psi=\{v \leqq w \mid P \subseteq Z(v)\}$ and define $C_{P}(w)$ to be the valuation with ring

$$
\mathscr{R}=\bigcup_{v \in \Psi} \mathscr{R}_{v}
$$

Then $a \in P$ implies that $a \in \mathscr{P}_{v}$ for all $v \in \Psi$, i.e., that $a^{-1} \notin \mathscr{R}_{v}$ for all $v \in \Psi$, and consequently $a^{-1} \notin \mathscr{R}$. It follows that $a \in \mathscr{P}$, i.e., that $P \subseteq \mathscr{P} \cap A$, and so $C_{P}(w) \in \Psi$, and is the coarsest of the valuations in $\Psi$.

Lemma 5. Let $\Omega$ be a family of finite character defining $A$.

Let $u_{0} \leqq w_{0} \in \Omega$ and $Q_{0}=Z\left(u_{0}\right)$.

Let $w^{\prime} \in \Omega_{+}\left(Q_{0}\right)$ with $v_{0}=C_{Q_{0}}\left(w^{\prime}\right)$.

Suppose that there exists a critical valuation $v_{0}^{*} \leqq v_{0}$ such that if $v_{0}^{*} \leqq v^{\prime} \leqq v_{0}$ then:

(1) $v^{\prime}$ is strongly well centered;

(2) $v^{\prime}$ is either critical or a limit valuation. Then we may conclude that $Z\left(v_{0}\right)=Q_{0}$.

\section{Proof.}

Case I. $v_{0}$ is not a limit valuation.

$v_{0}$ is critical at some $x$ and there exists a finest valuation strictly coarser than $v_{0}$; let this be $v_{1}$. Then $Q_{0} \nsubseteq Z\left(v_{1}\right)$, so there exists $a \in Q_{0}$ such that $a \notin Z\left(v_{1}\right)$, and since $v_{0}\left(a^{-1}\right)<0$, but $v_{1}\left(a^{-1}\right)=0$, it follows that $v_{0} \in \Sigma\left(a^{-1}\right)$. Since $v_{0}\left(x^{-1}\right)>0$, there exists an integer $n>0$ such that $v_{0}\left(a^{-1}\left(x^{-1}\right)^{n}\right)>0$, for otherwise $v_{0}(a)>n v_{0}\left(x^{-1}\right)$ for all $n$ and so $w^{\prime}\left(x^{-1}\right)$ belongs to the largest isolated subgroup of $\Gamma_{w^{\prime}}$ not containing $w^{\prime}\left(a^{-1}\right)$ but since $v_{0} \in \Sigma\left(a^{-1}\right)$ this implies $v_{0}\left(x^{-1}\right)=0$ in contradiction to $v_{0}\left(x^{-1}\right)>0$. It follows that for some $n, v_{0}\left(a x^{n}\right)<0$.

$v_{0}$ is critical at $a x^{n}:$ for $v_{0} \in \Sigma\left(a x^{n}\right)$, since $v_{0}\left(a x^{n}\right)<0$, and $v_{1}\left(a x^{n}\right)=v_{1}\left(x^{n}\right)=0$, and by Lemma 2 if $v \in \Sigma\left(a x^{n}\right)$ with $Z(v) \subseteq Z\left(v_{0}\right)$, then $Z(v)=Z\left(v_{0}\right)$.

Since $v_{0} \in M\left(a x^{n}\right), v_{0}$ is strongly well centred, and $Z\left(u_{0}\right)=Q_{0} \subseteq Z\left(v_{0}\right)$, by Lemma 4 it follows that $u_{0}\left(a x^{n}\right) \leqq 0$, i.e., $u_{0}\left(x^{n}\right) \leqq-u_{0}(a)<0$; hence $u_{0}(x)<0$. Again, $v_{0} \in M(x), v_{0}$ is strongly well centred, $Z\left(u_{0}\right) \subseteq Z\left(v_{0}\right)$ and $u_{0}(x)<0$; hence, by Lemma $4, Z\left(u_{0}\right)=Z\left(v_{0}\right)$, i.e., $Z\left(v_{0}\right)=Z\left(u_{0}\right)=Q_{0}$.

Case II. $v_{0}$ is a limit valuation and if $v^{\prime}$ belongs to the set $\varepsilon=\left\{v^{\prime} \mid v_{0}^{*} \leqq v^{\prime}<v_{0}\right.$, $v^{\prime}$ not a limit valuation $\}$ then $Z\left(v^{\prime}\right) \subseteq Q_{0}$. 
In this case, $Z\left(v_{0}\right)=\bigcup_{v^{\prime} \in \varepsilon} Z\left(v^{\prime}\right) \subseteq Q_{0}$ and from $Q_{0} \subseteq Z\left(v_{0}\right)$, it follows that $Z\left(v_{0}\right)=Q_{0}$.

Case III. $v_{0}$ is a limit valuation and there exists $v^{*}, v_{0}^{*} \leqq v^{*}<v_{0}$ such that $v^{*}$ is critical and $P^{*}=Z\left(v^{*}\right) \nsubseteq Q_{0}$.

If this case occurs, $Q_{0} \neq Z\left(v_{0}\right)$ (for otherwise $P^{*} \subseteq Z\left(v_{0}\right)=Q_{0}$ ).

We shall show that Case III cannot occur.

Let $n \geqq 0$ be an arbitrary integer. We shall prove that there exist valuations $w_{0}, w_{1}, \ldots, w_{n} \in \Omega$, which are pairwise nonequivalent and such that there exist valuations $u_{i} \leqq w_{i}$ such that $Z\left(v_{0}^{*}\right) \subseteq Z\left(u_{i}\right)=Q_{i}$ (for $i=0,1, \ldots, n$ ). Since $v_{0}^{*}$ is critical (hence nontrivial) $Z\left(v_{0}^{*}\right) \neq 0$ (because $\mathscr{R}_{v_{0}^{*}} \supseteq A_{Z\left(v_{0}^{*}\right)}$ ); let $a \in Z\left(v_{0}^{*}\right), a \neq 0$; then $u_{i}(a)>0$ and therefore $w_{i}(a)>0$ for all $i=0,1, \ldots, n$. Since $n$ is arbitrary, this contradicts the fact that $\Omega$ is a family of finite character.

Diagram of Prime Ideals

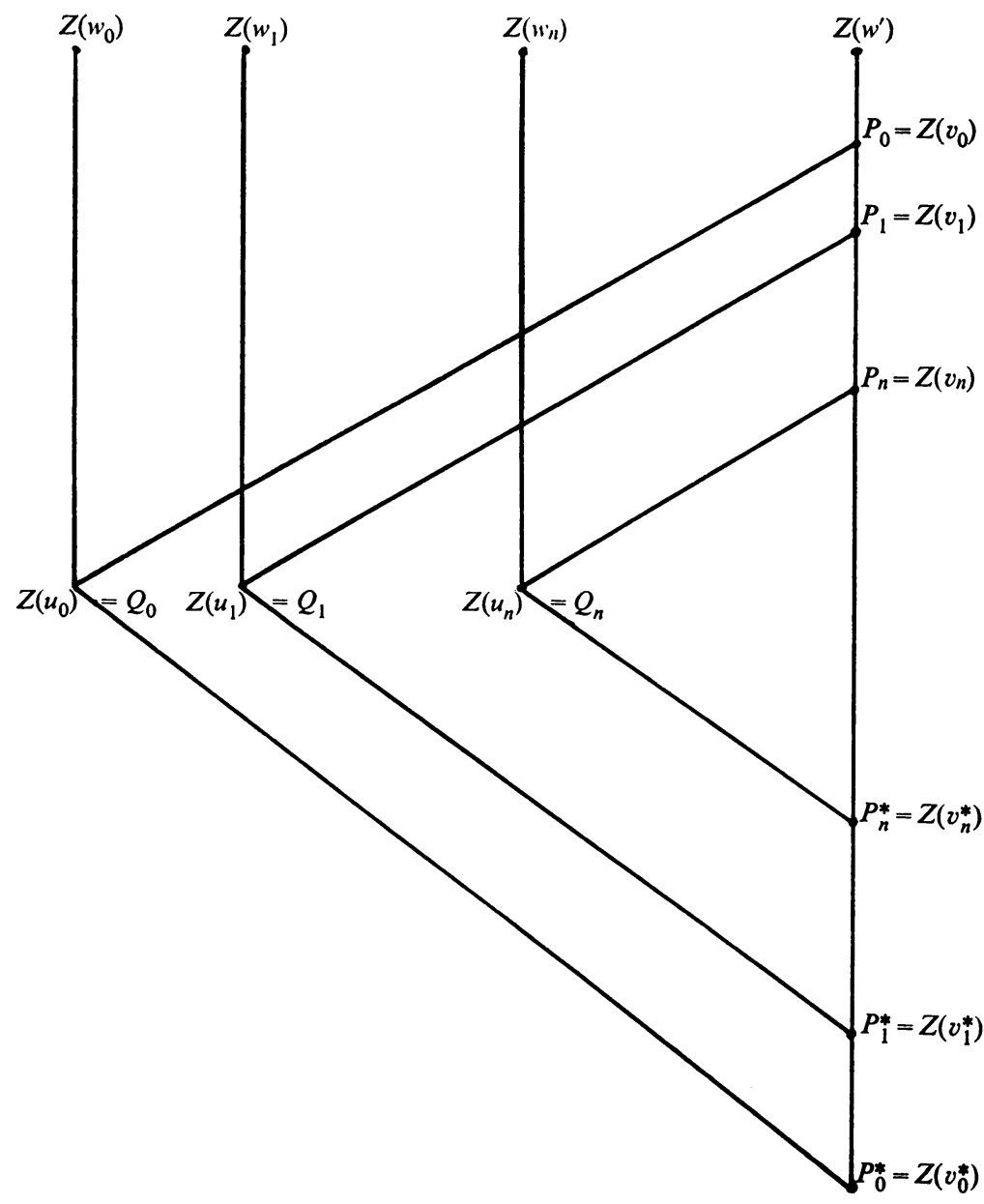


In order to prove the existence of the valuations $w_{i}, u_{i}$ as above, we shall proceed by induction on $n$. Precisely, for every $n \geqq 0$ we shall establish that there exist valuations $w_{i} \in \Omega, u_{i} \leqq w_{i}(i=0,1, \ldots, n)$ such that:

(1) the valuations $w_{i}$ are pairwise nonequivalent;

(2) $Z\left(u_{i}\right)=Q_{i} \subseteq Z\left(w^{\prime}\right)$ for $i=0,1, \ldots, n$;

(3) if $v_{i}=C_{Q_{i}}\left(w^{\prime}\right)$ and $P_{i}=Z\left(v_{i}\right)$ then $P_{i} \neq Q_{i}$ for $i=0,1, \ldots, n$;

(4) $v_{0} \geqq v_{1} \geqq \cdots \geqq v_{n} \geqq v_{0}^{*}$,

(5) for every $i=1, \ldots, n$, there exists a critical valuation $v_{i}^{*}$ such that $v_{i}>v_{i}^{*} \geqq v_{0}^{*}$ and $Z\left(v_{i}^{*}\right) \subseteq Q_{i}$ but $Z\left(v_{i}^{*}\right) \nsubseteq Q_{j}$ for $j=0,1, \ldots, i-1$.

For $n=0$, the hypothesis in Case III implies that (1) to (5) hold with $u_{0}, w_{0}, v_{0}$.

Let us assume that (1) to (5) hold for $n$ and let us determine $w_{n+1} \in \Omega, u_{n+1}$, $v_{n+1}^{*}$.

Consider $u_{n} \leqq w_{n} \in \Omega, v_{n}=C_{Q_{n}}(w)$ and $v_{n}^{*}$. We may substitute them for $u_{0}, v_{0}$ and $v_{0}^{*}$ in the hypothesis of the lemma. Since $Q_{n} \neq P_{n}$ we are already in Case III. Hence $v_{n}$ is a limit valuation and there exists $v_{n+1}^{*}$ which is critical at an element $x^{\prime} \in K$, $x^{\prime} \neq 0$, with $v_{n}^{*} \leqq v_{n+1}^{*}<v_{n}$ and $P_{n+1}^{*}=Z\left(v_{n+1}^{*}\right) \nsubseteq Q_{n}$. By induction, $P_{n}^{*}=Z\left(v_{n}^{*}\right) \nsubseteq Q_{j}$ (for $j=0,1, \ldots, n-1$ ), hence from $P_{n}^{*} \subseteq P_{n+1}^{*}$ (because $v_{n}^{*} \leqq v_{n+1}^{*}$ ) it follows that $P_{n+1}^{*} \nsubseteq Q_{j}($ for $j=0,1, \ldots, n-1, n)$.

By the corollary to Lemma 4 , since $P_{n+1}^{*}$ is a critical prime at $x^{\prime} \in K, x^{\prime} \neq 0$, and all valuations with centre $P_{n+1}^{*}$ are well centred, there exists $c \in K, c \neq 0$, such that if $x=c x^{\prime}$ then $\Omega^{-}(x)=\Omega_{0}\left(P_{n+1}^{*}\right)$. Since $v_{i}=C_{Q_{i}}\left(w^{\prime}\right)$, and $v_{i}>v_{n+1}^{*}$ then $Q_{i} \nsubseteq P_{n+1}^{*}$, for all $i=0,1, \ldots, n$; hence there exists an element $b \in \prod_{i=0}^{n} Q_{i}, b \notin P_{n+1}^{*}$. From $Q_{i}=Z\left(u_{i}\right), u_{i} \leqq w_{i}$, it follows that $w_{i} \notin \Omega_{0}\left(P_{n+1}^{*}\right)$ for $i=0,1, \ldots, n$ (because the set of valuations coarser than $w_{i}$ is totally ordered, and so are their centers on $A$, therefore none of these valuations could have center equal to $\left.P_{n+1}^{*}\right)$. We deduce that $w_{i}(x) \geqq 0$ for $i=0,1, \ldots, n$.

Therefore $u_{i}(x) \geqq 0$ and $u_{i}(b x)>0$ for $i=0,1, \ldots, n$, while $v_{n+1}^{*}(b x)<0$ (because $v_{n+1}^{*} \in \Omega_{0}\left(P_{n+1}^{*}\right)=\Omega^{-}(x)$ and $\left.v_{n+1}^{*}(b)=0\right)$, hence $v_{n}(b x)<0$. Since $v_{n}$ is well centred, there exists $a \in A$ such that $v_{n}(a)=-v_{n}(b x)>0$, so $v_{n}(a b x)=0$.

We show that there exists $u_{n+1} \in M(a b x)$ such that $Q_{n+1}=Z\left(u_{n+1}\right) \subseteq P_{n} \subseteq Z\left(w^{\prime}\right)$. If this were false, by Lemma 3 there exists $d \in A, d \notin P_{n}$ such that $d a b x \in A$. From $v_{n}(a b x)=0$ it follows that $v_{n}(d a b x)=0$, so $d a b x \notin P_{n}$; hence $d a b x \notin Q_{n} \subseteq P_{n}=Z\left(v_{n}\right)$, and therefore $u_{n}(d a b x)=0$. Since $d a \in A$ we conclude that $u_{n}(b x) \leqq 0$, which is a contradiction.

Let $w_{n+1} \in \Omega$ be such that $w_{n+1} \geqq u_{n+1}$. Then $w_{n+1}$ is not equivalent to $w_{i}$, because $w_{n+1}(b x) \leqq w_{n+1}(a b x)<0$ while $w_{i}(b x) \geqq 0$ (for $\left.i=0,1, \ldots, n\right)$.

Now, we show that $Q_{n+1} \supseteq P_{n+1}^{*}$. Since $w_{n+1} \in \Omega^{-}(x)=\Omega_{0}\left(P_{n+1}^{*}\right)$ there exists a valuation $u^{\prime}$ of $K, u^{\prime} \leqq w_{n+1}$, such that $Z\left(u^{\prime}\right)=P_{n+1}^{*}$. If $u^{\prime} \leqq u_{n+1}$ then $P_{n+1}^{*} \subseteq Q_{n+1}$. Let us assume that $u_{n+1} \leqq u^{\prime}$, hence $Q_{n+1} \subseteq P_{n+1}^{*}$; noting that $P_{n+1}^{*}$ is a critical prime at $x^{\prime}$ and $u_{n+1} \in M\left(a b c x^{\prime}\right)$, it follows from Lemma 2 that $Z\left(u_{n+1}\right)=P_{n+1}^{*}$.

Let $v_{n+1}=C_{Q_{n+1}}\left(w^{\prime}\right)$; from $Q_{n+1} \subseteq P_{n}$ it follows that $v_{n+1} \leqq v_{n}$. If $Q_{n+1}=P_{n+1}$ $=Z\left(v_{n+1}\right)$, then by Lemma 4 we have $v_{n+1}(a b x)<0$ (because $u_{n+1} \in M(a b x)$ and 
$u_{n+1}$ is strongly well centred, since the same holds for $v_{n+1}, v_{0}^{*} \leqq v_{n+1} \leqq v_{0}$, by hypothesis); thus $v_{n}(a b x)<0$ and this is a contradiction. Therefore $Q_{n+1}$ is strictly contained in $P_{n+1}$, hence $P_{n+1}^{*} \subset P_{n+1}$ hence $v_{n+1}^{*}<v_{n+1}$.

This concludes the induction, proving the lemma.

Proposition 6. Let $\Omega$ be a family of finite character defining A. Let $P$ be a critical prime such that all valuations in $\omega_{-}(P)$ are well centred and either critical or limit valuations.

Then $A_{P}=\mathscr{R}_{v}$ for every $v \in \omega_{0}(P)$.

Proof. Let $v \in \omega_{0}(P)$ and let $w \in \Omega$ be such that $v \leqq w$. We need to show that if $a, b \in A$ with $v(a) \geqq v(b)$, then there exists $c \in K$ such that $b c, a c \in A$ and $v(b c)=0$.

Let $x \in K$ be such that $v(x)=-v(b)$.

We use Lemma 5 to show that if $u_{0} \in M(b x)$ then $Z\left(u_{0}\right) \nsubseteq P$.

For suppose that $u_{0} \in M(b x)$ with $Z\left(u_{0}\right) \subseteq P, u_{0} \leqq w_{0} \in \Omega$. Then $Z\left(u_{0}\right)=Q_{0}$ $\subseteq Z(v)=P \subseteq Z(w)$. Let $v_{0}=C_{Q_{0}}(w)$, hence $v_{0} \leqq v$. If $v_{0}$ is not a limit take $v_{0}^{*}=v_{0}$; otherwise take $v_{0}^{*}$ equal to any valuation $v^{\prime} \leqq v_{0}$ which is nontrivial and not a limit. Then $v_{0}^{*} \leqq v^{\prime} \leqq v_{0}$ implies that $v^{\prime} \in \omega_{-}(P)$ so $v^{\prime}$ is strongly well centred and either critical or limit. Thus by Lemma $5, Z\left(v_{0}\right)=Z\left(u_{0}\right)=Q_{0} \subseteq P$. Now since $u_{0}$ is critical at $b x$ and strongly well centred it follows from Lemma 4 that $v_{0}(b x)<0$; hence $v(b x)<0$, a contradiction.

Thus $u_{0} \in M(b x)$ implies $Z\left(u_{0}\right) \nsubseteq P$ and by Lemma 3 there exists $d \in A, d \notin P$ such that $d b x \in A, v(d b x)=v(b x)=0$.

Now $v(d a x) \geqq v(d b x)=0$ and as above, if $u_{0} \in M($ dax $)$ with $Z\left(u_{0}\right) \subseteq P$, by Lemma 4 it follows that $Z\left(u_{0}\right)=Z\left(v_{0}\right)$ with $v_{0} \leqq v$, and so by Lemma $4, v_{0}($ dax $)<0$, in contradiction to $v($ dax $) \geqq 0$.

Thus $u_{0} \in M($ dax $)$ implies $Z\left(u_{0}\right) \nsubseteq P$ and by Lemma 3 there exists $e \in A, e \notin P$ such that edax $\in A$, and $v(e d a x)=v($ dax $) \geqq 0$. Also $v(e d b x)=v(b d x)=0$ so we may take $c=e d x$.

THEOREM 7. Let $\Omega$ be a family of finite character defining $A$ such that every valuation of $\Omega$ is well centered and uniformly critical for A. Then A is a ring of Krull type, such that every valuation $w \in \Omega$ is essential for $A$.

Proof. This follows immediately from Proposition 6.

\section{Further results on essential valuations.}

Proposition 8. Let $\Omega$ be a family of finite character consisting of rank one valuations, with all critical valuations well centered. Then $\Omega$ defines a ring of Krull type A.

Proof. Let $\Omega^{\prime}$ be those valuations of $\Omega$ which are critical (with respect to $\Omega$ ). Let $A^{\prime}=\bigcap_{w \in \Omega^{\prime}} \mathscr{R}_{w}$, hence $A \subseteq A^{\prime}$. On the other hand, if $x \notin A$, then for some $w \in \Omega, w \in M(x)$ (since the valuations have rank one), so that $w \in \Omega^{\prime}$ and $x \notin A^{\prime}$. 
This shows that $A^{\prime}=A$. Now, $\Omega^{\prime}$ is a family of finite character in which each valuation is critical and well centered, but since the valuations of $\Omega^{\prime}$ are of rank one they are also uniformly critical. We conclude by Theorem 7 that $\Omega^{\prime}$ is an essential family of valuations and so $A$ is a ring of Krull type.

Ribenboim [5] has studied the rings which satisfy Proposition 8.

We say that a family of valuations $\Omega^{\prime}$ is coarser than $\Omega$ if every valuation $w^{\prime} \in \Omega^{\prime}$ is coarser than some valuation $w$ of $\Omega$.

Proposition 9. Let $\Omega$ be a family of finite character. Let $S, 0 \notin S$, be a multiplicatively closed subset of $A$.

Let $\mathscr{E}$ be the family of prime ideals of $A$ having empty intersection with $S$, and assume that each prime in $\mathscr{E}$ contains a minimal prime in $\mathscr{E}$, and that there are only a finite number of minimal primes in $\mathscr{E}$.

Then $A_{S}$ is the intersection of a finite number of valuation rings.

Proof. We use a result from [1]: there exists a family of valuations $\Omega^{\prime}$, coarser than $\Omega$, such that $A_{S}=\bigcap_{w \in \Omega^{\prime}} \mathscr{R}_{w}$. Since $A_{S}$ has only finitely many minimal prime ideals, their product is nonzero, hence every nonzero element in this product belongs to the centre of every valuation of $\Omega^{\prime}$; because of the finite character of $\Omega^{\prime}$, we conclude that $\Omega^{\prime}$ must be a finite family.

For any prime ideal $P$ of $A$, let $\mathscr{E}(P)$ denote the set of prime ideals of $A$ contained in $P$. If $w$ is an essential valuation for $A$, then $\mathscr{E}(Z(w))$ is totally ordered. In fact, $A_{Z(w)}=\mathscr{R}_{w}$, by hypothesis; since there is a one-to-one inclusion-preserving mapping between $\mathscr{E}(Z(w))$ and the set of prime ideals of $A_{Z(w)}$, it follows that $\mathscr{E}(Z(w))$ is totally ordered by inclusion.

Proposition 10. Let $\Omega$ be a family of finite character defining $A$ and suppose that every prime ideal of $A$ contains a minimal prime ideal. If $\mathscr{E}(Z(w))$ is totally ordered for every valuation $w \in \Omega$ then $\Omega$ is a family of essential valuations for $A$.

Proof. Let $\mathscr{E}(Z(w))$ be totally ordered for each $w \in \Omega$. Then $Z(w)$ contains just one minimal prime ideal and by Proposition 9, applied to the complement of $Z(w)$, $A_{Z(w)}$ is the intersection of a finite number of valuations rings. Now Ribenboim [6] has shown that a local ring which is the intersection of a finite family of valuation rings is a valuation ring; hence $A_{Z(w)}$ is a valuation ring and we conclude that $w$ is an essential valuation.

We now define the finest valuation which is coarser than a given valuation $w$, and has centre contained in a prime $P$ of $A$ with $A \subseteq \mathscr{R}_{w}$. We shall denote this valuation $F_{P}(w)$. Define $\mathscr{E}=\left\{v^{\prime} \leqq w \mid Z\left(v^{\prime}\right) \subseteq P\right\}$; then $\mathscr{E}$ is not empty since it contains the trivial valuation. Define $F_{P}(w)$ to be the valuation with ring

$$
\mathscr{R}=\bigcap_{v^{\prime} \in \mathscr{E}} \mathscr{R}_{v^{\prime}}
$$

To show that $F_{P}(w)$ is the finest valuation belonging to $\mathscr{E}$, we need only show that $Z\left(F_{P}(w)\right) \subseteq P$. If $a \in Z\left(F_{P}(w)\right)$, then $a$ belongs to the maximal ideal of $\mathscr{R}$; so $a^{-1} \notin \mathscr{R}$ 
and hence for some $v^{\prime} \in \mathscr{E}, a^{-1} \notin \mathscr{R}_{v^{\prime}}$, i.e., $a \in P_{v^{\prime}}$, and $a \in Z\left(v^{\prime}\right) \subseteq P$; thus $Z\left(F_{P}(w)\right)$ $\subseteq P$.

LEMMA 11. Let $\Omega$ be a family of finite character. Let $P$ be a prime ideal which is such that $\mathscr{E}(P)$ is totally ordered. Then there exists a valuation $v^{\prime}$, coarser than some valuation $w^{\prime} \in \Omega$ such that $Z\left(v^{\prime}\right)=P$.

Proof. If the trivial valuation is the only element of $\omega_{-}(P)$, let $v^{\prime}$ be the trivial valuation.

If there exists a nontrivial valuation $v_{0}$ of $K, v_{0} \in \omega_{-}(P)$, then $Z\left(v_{0}\right) \neq 0$ and therefore the set $\Omega_{+}\left(Z\left(v_{0}\right)\right)$ is finite, because $\Omega$ is a family of finite character. For every $w \in \Omega$ such that $Z(w) \supseteq Z\left(v_{0}\right)$, we consider the valuation $F_{P}(w)$; since $\mathscr{E}(P)$ is totally ordered, and $\Omega_{+}\left(Z\left(v_{0}\right)\right)$ is finite, there exists a valuation $w^{\prime} \in \Omega_{+}\left(Z\left(v_{0}\right)\right)$ such that $Z\left(F_{P}\left(w^{\prime}\right)\right)$ is the largest possible; we take $v^{\prime}=F_{P}\left(w^{\prime}\right)$. Clearly, $Z\left(v_{0}\right) \subseteq Z\left(v^{\prime}\right)$.

Now, we prove that $Z\left(v^{\prime}\right)=P$. If $Z\left(v^{\prime}\right) \neq P$ then $Z\left(v^{\prime}\right) \subset P$ and there exists $x \in P$, $x \notin Z\left(v^{\prime}\right)$. Then, if $v \in \Sigma\left(x^{-1}\right)$ it follows that $Z(v) \nsubseteq P$. In fact, if $Z(v) \subseteq P$, since $\mathscr{E}(P)$ is totally ordered, either $Z(v) \subseteq Z\left(v_{0}\right)$ or $Z\left(v_{0}\right) \subseteq Z(v)$; in the first case, from $x \in P$, $v(x)>0$ it follows that $v_{0}(x)>0$; hence also $v^{\prime}(x)>0$, which is a contradiction; if $Z\left(v_{0}\right) \subseteq Z(v)$ and if $w \in \Omega$ is such that $v \leqq w$, then by the choice of $v^{\prime}$, we have $Z(v) \subseteq Z\left(F_{P}(w)\right) \subseteq Z\left(v^{\prime}\right)$; therefore from $v(x)>0$ we conclude again that $v^{\prime}(x)>0$, a contradiction. Since $v \in \Sigma\left(x^{-1}\right)$ implies $Z\left(v_{0}\right) \nsubseteq P$ it follows by Lemma 3 that there exists $a \in A, a \notin P$, such that $a x^{-1} \in A$. Then $a=a x^{-1} x \in P$, a contradiction. We conclude that $Z\left(v^{\prime}\right)=P$.

Proposition 12. Let $\Omega$ be a family of finite character defining the ring $A$. Let $v$ be an essential valuation of $A$. Then there exists $w \in \Omega$ with $w \geqq v$.

Proof. Since $v$ is an essential valuation for $A, \mathscr{E}(Z(v))$ is totally ordered; hence by Lemma 11 there exists $w \in \Omega$ with $v^{\prime} \leqq w \in \Omega$ and $Z\left(v^{\prime}\right)=Z(v)$; from $\mathscr{R}_{v}=A_{Z(v)} \subseteq \mathscr{R}_{v^{\prime}}$, Lemma 1 and the remark following it, we deduce that $v$ and $v^{\prime}$ are equivalent and $w \geqq v$.

Lemma 11 may be used to prove the following two results. In the first, which deals with $t$-ideals, we use the following globalization lemma, proved in [1]; if $A$ is a ring of Krull type defined by the family $\Omega$ of finite character, consisting of essential valuations, and if $M$ is any integral ideal of $A$, then $M_{t}=\bigcap_{w \in \Omega} \mathscr{R}_{w} M$.

Proposition 13. Let $M$ be an integral ideal of a ring of Krull type A. Then $M_{t} \neq A$ if and only if there exists a prime $P$ with $M \subseteq P$ such that $\mathscr{E}(P)$ is totally ordered.

Proof. If $M_{t}=\bigcap_{w \in \Omega} \mathscr{R}_{w} M \subset A$, then for some $w \in \Omega, \mathscr{R}_{w} M \cap A \subset A$ so $M \subseteq Z(w)$ and since $w$ is essential, $\mathscr{E}(Z(w))$ is totally ordered.

If $M \subseteq P$ with $\mathscr{E}(P)$ totally ordered, then by Lemma 11 there exists $v \leqq w \in \Omega$ with $Z(v)=P$ and consequently $M_{t}=\bigcap_{w \in \Omega} \mathscr{R}_{w} M \subseteq \mathscr{R}_{w} Z(w) \cap A=Z(w) \subset A$ (because $w \in \Omega$ is an essential valuation for $A$ ). 
Proposition 14. Let $A$ be a ring of Krull type; then $A$ is a Prüfer ring if and only if $\mathscr{E}(P)$ is totally ordered for every maximal ideal $P$.

Proof. If $A$ is a Prüfer ring then $A_{P}$ is an essential valuation with centre $P$ and $\mathscr{E}(P)$ is a totally ordered set.

Let $\Omega$ be an essential family of finite character defining $A$. Since $\mathscr{E}(P)$ is totally ordered, by Lemma $11, P$ is the centre of a valuation $v \leqq w \in \Omega$; hence $Z(v)=P$ $\subseteq Z(w)$; since $P$ is a maximal ideal of $A$, it follows that $v=w$. Now $w$ is essential, so $A_{P}$ is a valuation ring and since this holds for every maximal ideal $P, A$ is a Prüfer ring.

\section{Examples.}

1. A ring, not of Krull type, which is defined by a family of finite character consisting of rank one valuations. Another example of such a ring has also been found independently by $\mathrm{Ohm}[4]$.

Let $k$ be a field.

We define the valuation $w$ on $k[X, Y]$ by setting $w\left(\sum_{i=1}^{n} a_{i} X^{n_{i}} Y^{m_{i}}\right)=\min _{1 \leqq i \leqq n}$ $\left\{(2)^{1 / 2} n_{i}-m_{i}\right\}$; the valuation is extended to $k(X, Y)$ by putting $w(a / b)=w(a)-w(b)$ when $a, b \in k[X, Y]$. It is not difficult to show that $w$ is a rank one valuation; a proof may be found in [7, p. 100].

Let $\Omega$ be the family of valuations $v_{p}$ of $k(X, Y)$ defined by irreducible polynomials $p$ of $k[X, Y]$. Then $\Omega$ has finite character and defines $k[X, Y]$.

Let $\Omega^{\prime}=\Omega \cup\{w\}$; then $\Omega^{\prime}$ has finite character, and since $w(Y)<0, \Omega^{\prime}$ defines a ring $A$ properly contained in $k[X, Y]$ for which $w$ is a critical valuation.

Now if $w$ were well centred then there would exist an element $a \in A$ such that $w(a)=1$. Since $A \subseteq k[X, Y]$, we have $a=p(X, Y)$, for some polynomial $p(X, Y)$; hence, there would exist positive integers $n, m$, such that $n(2)^{1 / 2}-m=1$, i.e., $(2)^{1 / 2}=(m+1) / n$, which is clearly impossible.

Suppose that $A$ is a ring of Krull type. Then since $A \subset k[X, Y]$, there must be some essential valuation $v$ of $A$ not contained in $\Omega$. By Proposition 12 we must have $w^{\prime} \geqq v$ for some $w^{\prime} \in \Omega^{\prime}$; the only possible candidate is $w$, and since $w$ is of rank one, $w=v$. Thus $w$ is essential and hence well centred, a contradiction. We conclude that $A$ is not a ring of Krull type.

This counterexample, together with Proposition 8 , provides a solution to the question posed by Krull in [6]. The counterexample also shows that the assumption that the valuations were well centred in Theorem 7 is necessary.

2. A ring, not of Krull type, defined by a well centred family of finite character. Let $\Omega$ be the family of valuations defined by all the irreducible polynomials of $k[X, Y, Z]$, except the polynomial $Z$.

Define $v$ on $k[X, Y]$ by setting $v(p(X, Y))=m$, if $p(X, Y)$ has terms of total degree $m$, but none of lower degree. Extend $v$ to $k(X, Y)$ by setting $v(f / g)=v(f)$ $-v(g)$. Then $v$ is the $(X, Y)$-adic divisor of $k(X, Y)$ [7, p. 105]. $v$ may be extended canonically to $k(X, Y, Z)$, and as a valuation of $k(X, Y, Z)$ it will have residue 
field $k(Z)$. Let $v^{\prime}$ be the valuation of $k(Z)$ defined by the minimal polynomial $Z$. Let $w=v^{\prime} \cdot v$ be the corresponding discrete rank two valuation of $k(X, Y, Z)$. Let $\Omega^{\prime}$ be the family $\Omega \cup\{w\}$.

Let $A=\bigcap_{w \in \Omega^{\prime}} \mathscr{R}_{w}$. Then $A$ is easily seen to consist of all elements of the form $p_{1}(X, Y, Z) X Z^{-n}+p_{2}(X, Y, Z) Y Z^{-m}+p_{3}(Z) \quad$ where $p_{1}(X, Y, Z), \quad p_{2}(X, Y, Z) \in$ $k[X, Y, Z]$ and $p_{3}(Z) \in k[Z]$.

Now if $A$ were a ring of Krull type there would be some essential valuation $u$ of $A$, not contained in $\Omega$ (since $\Omega$ defines the ring of all elements of the form $\left.p(X, Y, Z) Z^{-n}\right)$; by Proposition 12 we must have $w \geqq u$, so that certainly $v$, the extension of the $(X, Y)$-adic divisor, will be an essential valuation.

If $v$ is essential then we must have either $X / Y \in \mathscr{R}_{v}=A_{(X, Y)}$ or $Y \mid X \in \mathscr{R}_{v}$ $=A_{(X, Y)}$ and we may suppose the former to be the case. It follows that there exists some element $b$ of $k(X, Y, Z)$ such that $b Y \in A, b Y \notin(X, Y) A$ and $b X \in A$. Since $b Y \notin(X, Y) A$, we must have

$$
b Y=p_{1}(X, Y, Z) X Z^{-n}+p_{2}(X, Y, Z) Y Z^{-m}+p_{3}(Z),
$$

where $p_{3}(Z) \neq 0$ (for otherwise $b Y \in(X, Y) A$ ). So $b X=p_{1}(X, Y, Z) X^{2} Z^{-n} Y^{-1}$ $+p_{2}(X, Y, Z) Z^{-m} X+p_{3}(Z) X Y^{-1}$ and $p_{3}(Z) X Y^{-1}$ cannot cancel with the first term because of the difference in the powers of $X$ involved. Thus we conclude $b X \notin A$, a contradiction. Therefore, $A$ is not a ring of Krull type.

Now since the valuations in $\Omega$ are essential for $k[X, Y, Z]$, they are essential for $A \supseteq k[X, Y, Z]$. Also, $w$ is well centred (as may be seen by taking elements $X^{n} Z^{m} \in A$ with $n \geqq 0$, and $m \geqq 0$ if $n=0$ ). It follows from Theorem 7 that $A$ must fail to be a ring of Krull type because $w$ fails to be uniformly critical. It is not hard to check that $w$ is not uniformly critical.

\section{REFERENCES}

1. Malcolm Griffin, Some results on v-multiplication rings, Canad. J. Math. 97 (1967), $710-722$.

2. - Rings of Krull type, J. Reine Angew. Math. (to appear).

3. Wolfgang Krull, Uber die Zerlengung der Hauptideale in allgemeinen Ringen, Math. Ann. 105 (1931), 1-14.

4. Jack Ohm, Some counter examples related to the integral closure in $D \llbracket X \rrbracket$, Trans. Amer. Math. Soc. 122 (1966), 321-333.

5. Paulo Ribenboim, Anneaux normaux réels à caractère fini, Summa Brasil. Math. 3 (1956), 213-253.

6. —_ Le théorème d'approximation pour les valuations de Krull, Math. Z. 68 (1957), 1-18.

7. O. Zariski and P. Samuel, Commutative algebra, Vol. II, Van Nostrand, Princeton, N. J., 1960.

Department of Scientific ANd Industrial Research, Wellington, New Zealand 